\title{
The Ability to Diagnose Intrahepatic Cholangiocarcinoma Definitively Using Novel Branched DNA-Enhanced Albumin RNA In Situ Hybridization Technology
}

\author{
Cristina R. Ferrone ${ }^{1}$, David T. Ting ${ }^{2}$, Mohammed Shahid ${ }^{1,3}$, loannis T. Konstantinidis ${ }^{1}$, \\ Francesco Sabbatino ${ }^{1}$, Lipika Goyal ${ }^{2}$, Travis Rice-Stitt ${ }^{3}$, Ayesha Mubeen ${ }^{1}$, Kshitij Arora ${ }^{1,3}$, \\ Nabeel Bardeesey², John Miura ${ }^{4}$, T. Clark Gamblin ${ }^{4}$, Andrew X. Zhu², Darrell Borger ${ }^{1}$, Keith \\ D. Lillemoe ${ }^{1}$, Miguel N. Rivera ${ }^{3}$, and Vikram Deshpande ${ }^{3}$ \\ Cristina R. Ferrone: CFERRONE@mgh.harvard.edu; Ioannis T. Konstantinidis: vikramdirdeshpande@gmail.com; Vikram \\ Deshpande: vdeshpande@partners.org \\ ${ }^{1}$ Department of Surgery, Massachusetts General Hospital, Boston, MA \\ 2Division of Hematology/Oncology, Department of Medicine, Massachusetts General Hospital, \\ Boston, MA \\ ${ }^{3}$ Division of Pathology, Massachusetts General Hospital, Boston, MA \\ ${ }^{4}$ Division of Surgical Oncology, Medical College of Wisconsin, Milwaukee
}

\begin{abstract}
Background-Intrahepatic cholangiocarcinoma (ICC) often is a diagnosis determined by exclusion. Distinguishing ICC from other metastatic adenocarcinomas based on histopathologic or immunohistochemical analysis often is difficult and requires an extensive workup. This study aimed to determine whether albumin, whose expression is restricted to the liver, has potential as a biomarker for ICC using a novel and highly sensitive RNA in situ hybridization (ISH) platform.
\end{abstract}

Methods-Modified branched DNA probes were developed for albumin RNA ISH. The study evaluated 467 patient samples of primary and metastatic lesions.

Results-Of the 467 samples evaluated, 83 were ICCs, 42 were hepatocellular carcinomas (HCCs), and 332 were nonhepatic carcinomas including tumors arising from the perihilar region and bile duct, pancreas, stomach, esophagus, colon, breast, ovary, endometrium, kidney, and urinary bladder. Albumin RNA ISH was highly sensitive for cancers of liver origin, staining positive in $82(99 \%)$ of 83 ICCs and in $42 \mathrm{HCCs}(100 \%)$. Perihilar and distal bile duct carcinomas as well as carcinomas arising at other sites tested negative for albumin. Notably, 6 (22 $\%$ ) of 27 intrahepatic tumors previously diagnosed as carcinomas of undetermined origin tested positive for albumin.

Conclusions-Albumin RNA ISH is a sensitive and highly specific diagnostic tool for distinguishing ICC from meta-static adenocarcinoma to the liver or carcinoma of unknown origin. Albumin RNA ISH could replace the extensive diagnostic workup, leading to timely confirmation

Electronic supplementary material The online version of this article (doi:10.1245/s10434-014-4247-8) contains supplementary material, which is available to authorized users. 
of the ICC diagnosis. Additionally, the assay could serve as a guide to distinguish ICC from perihilar adenocarcinoma.

The diagnosis of intrahepatic cholangiocarcinoma (ICC) is one of the most difficult for a clinician to make when a patient presents with a newly identified hepatic mass. Although ICC is the second most common primary liver cancer, this neoplasm continues to be a diagnosis determined by exclusion. ${ }^{1,2}$ Our ability to make the diagnosis has not improved over the last several decades, although the incidence of ICC has been steadily increasing.

Usually, ICC presents as a solitary lesion, but it also can present as multiple intrahepatic lesions. ${ }^{3,4}$ Only subtle histopathologic differences exist between ICC and meta-static adenocarcinoma to the liver, making the diagnosis of ICC difficult. Pathologists most often resort to evaluating keratin profiles of the tumor including keratin 7, keratin 19, and keratin 20. Unfortunately, a majority of metastatic adenocarcinomas share this keratin $7+$, keratin $19+$, and keratin 20- profile, making immunohistochemistry nondiagnostic.

Surgeons, medical oncologists, and radiation oncologists generally require a definitive diagnosis before rendering a treatment decision. Because pathologic examination with immunohistochemistry often is unable to distinguish primary adenocarcinoma from metastatic cancer, patients must undergo an exhaustive evaluation to exclude a potential primary carcinoma at other sites. ${ }^{3}$ Blood work also is not very helpful given that the commonly used tumor markers [i.e., cancer antigen (CA) 19-9, carcino-embryonic antigen, a-fetoprotein, and CA125] are not specific to ICC. ${ }^{5}$ Therefore, patients are subjected to upper gastrointestinal endoscopy to rule out a gastric or duodenal source and colonoscopy to rule out a colorectal primary. Other tests often are considered include a cervical pap smear, mammogram, computed tomography, magnetic resonance imaging, and positron emission tomography.

Only when many or all of these examinations show negative results do physicians often feel comfortable making the diagnosis of ICC. Unfortunately, these studies not only take time but also place patients at risk for procedure-related complications. The cost of these tests to the health care system also is significant. A sensitive and specific marker for the diagnosis of ICC is urgently needed to improve the care of patients.

We identified albumin as a potential diagnostic marker of ICC given its abundant and highly specific RNA expression in cells of hepatocyte origin. ${ }^{6-9}$ Therefore, we evaluated albumin RNA on paraffin-embedded tissue using branched-DNA-based probes. Branched-DNAbased RNA in situ hybridization (ISH) has distinct advantages in the evaluation of Formalin fixed paraffin embedded (FFPE) tissue:

1. Immunohistochemistry for albumin demonstrates a strong nonspecific background signal due to the ubiquitous presence of albumin in serum.

2. Using existing ISH platforms, albumin mRNA is expressed in hepatocellular carcinomas (HCCs). ${ }^{7,8,10,11}$

3. The novel RNA ISH platforms are more sensitive and specific than traditional platforms. 
This study aimed to use this novel branched-DNA platform to evaluate albumin RNA ISH as a sensitive and specific diagnostic marker for ICC.

\section{PATIENTS AND METHODS}

\section{Study Cases}

Clinical and pathology databases were used to identify cases of adenocarcinoma diagnosed at the Massachusetts General Hospital (MGH) between January 2004 and December 2013. Formalin-fixed specimens of ICC, HCC, and normal liver cases were selected. The distinction of ICC from perihilar carcinomas was based on evaluation of the surgical specimen, imaging, and the operative report. Primary adenocarcinomas and the corresponding liver metastases evaluated included tumors arising in the lung $(n=22)$, esophagus and gastroesophageal junction $(n=40)$, stomach $(n=72)$, colon $(n=40)$, pancreas $(n=210)$, renal cells $(n=46)$, breast $(n=34)$, bladder $(n=8)$, endometrium $(n=$ $8)$, and ovary $(n=6)$, representing the most common carcinomas that metastasize to the liver.

All types of tumor were placed on tissue microarrays constructed using 2-mm cores of paraffin-embedded tissue. Entire sections of 30 ICC and 30 pancreatic ductal adenocarcinomas were evaluated. An additional cohort of ICCs from the Medical College of Wisconsin also were evaluated. In addition to the known tumor types, 27 tumors of unknown origin were evaluated.

This study was approved by the institutional review boards at the MGH and the Medical College of Wisconsin.

\section{RNA ISH Platform}

In this study, ISH was performed with ViewRNA technology (Affymetrix, Santa Clara, CA, UDA) using manual and automated platforms (see Supplementary Material 1 for automated platform). The basis for View-RNA ISH assays is branched-DNA technology wherein signal amplification is achieved via a series of sequential hybridization steps (Fig. 1). A probe is composed of multiple oligonucleotides. The gene-specific oligonucleotide pairs hybridize to the target RNA. A signal amplification structure is created via serial hybridization steps in which a single preamplifier molecule hybridizes to each oligonucleotide probe pair. Multiple amplifier (AMP) molecules then hybridize to each preAMP, and multiple alkaline phosphatase-conjugated-label probes hybridize to each amplifier. Each fully assembled signal amplification structure has approximately 400 binding sites for the AP-labeled probe. After addition of the fast-red substrate, AP catalyzes the substrate to form a red dot or diffuse precipitate that allows detection of RNA transcripts in situ.

For these experiments, the albumin ISH probe (Affymetrix, Santa Clara, CA, USA) was designed using the National Center for Biotechnology Information (NCBI) nucleotide database (see Supplementary Data 2). Cases that lacked reactivity for albumin were stained with glyceral-dehyde phosphate dehydrogenase, a ubiquitously expressed gene, to assess the integrity of mRNA. Normal liver tissue served as a positive control substance. Mesenchymal cells and lymphoid cells served as negative control substances. Replicates were examined in 
21 ICC cases, with no differences in either the intensity or the distribution of stain.

Automation analysis was performed on the BondRx platform (Leica Biosystem, Buffalo

Grove, IL, USA) (also see Supplementary Material 1).

\section{Quantitative Analysis}

The specificity of the signal allowed for semiquantitative analysis, and the percentage of positive tumor cells was recorded at intervals of $5 \%$.

\section{RESULTS}

A total of 462 paraffin-embedded samples were stained for albumin via RNA ISH. Normal liver, HCC, extrahepatic biliary tract carcinomas, and ICCs were evaluated. Primary adenocarcinomas and metastatic liver lesions arising from pancreas, stomach, esophagus, colon, breast, ovary, endometrium, and bladder were evaluated with the albumin RNA ISH probe.

\section{Albumin RNA ISH Staining of Normal Liver}

To assess the prevalence of albumin as a specific marker of liver origin, 32 normal liver specimens were stained. Hepatocytes were strongly positive for albumin, whereas Kupffer cells, endothelial cells, fibroblasts, and stroma did not stain. Only a few interlobular bile ducts showed positive test results for albumin. However, reactive bile ductules in the setting of chronic hepatitis or biliary disease consistently tested positive for albumin.

\section{Albumin RNA ISH of Hepatocellular Carcinoma}

All 42 resected HCCs stained positively for albumin regardless of tumor grade. Findings showed 8 tumors to be well differentiated, 22 to be moderately differentiated, and 12 to be poorly differentiated, yet all demonstrated a diffuse expression pattern (Fig. 2).

\section{Albumin RNA ISH of ICC}

A total of 83 resected ICC cases from MGH and the Medical College of Wisconsin were stained with albumin RNA ISH. The median age of the patients was 65 years. The median tumor size was $5.5 \mathrm{~cm}$, and $18.2 \%$ of the patients had positive lymph nodes. Microvascular invasion was identified in 29 cases $(52.7 \%)$. An R0 resection was achieved for $49(89.1 \%)$ of 55 resected patients. The median overall survival period for the MGH cohort was 73 months.

Of the 83 cases from MGH and the Medical College of Wisconsin, 82 ICCs (99\%) tested positive for albumin (Fig. 3). The majority of the tumors (79\%) demonstrated reactivity in more than $50 \%$ of the tumor cells (Fig. 3). The pattern of reactivity generally was similar to that seen in HCC, but notably, the signal intensity per tumor cell in ICCs was less than that seen in HCCs. The positively stained tumors showed heterogeneity of staining, with reactivity ranging from 5 to $100 \%$ of tumor cells. Staining intensity did not correlate significantly with overall survival. 


\section{Extrahepatic Bile Duct Carcinomas}

Extrahepatic bile duct carcinomas appear histologically similar to ICC, but it is well known that the natural histories of these diseases are quite distinct. Consistent with these differences, albumin RNA ISH was completely negative in both perihilar adenocarcinomas $(n=24)$ and mid-distal bile duct adenocarcinomas $(n=22)$.

\section{Albumin RNA ISH of Other Carcinomas}

Clinically, the most difficult diagnostic problem has been the differentiation of ICC from metastatic adenocarcinoma to the liver. Therefore, we tested various types of metastatic adenocarcinomas with albumin RNA ISH. The primary adenocarcinoma and metastases from the lung $(n=22)$, pancreas $(n=210)$, esophagus and gastro-esophageal junction $(n=$ $40)$, stomach $(n=72)$, colon $(n=40)$, bladder (transitional cell carcinoma) $(n=8)$, ovary $(n$ $=6)$, endometrium $(n=8)$, renal cells $(n=46)$, and breast $(n=34)$ were evaluated. All the tumors tested negative for albumin.

\section{Tumors of Unknown Origin}

Tumors of unknown origin also present a significant clinical dilemma because the diagnosis is based on exclusion, and treatment regimens have not been established. We evaluated 27 intrahepatic adenocarcinomas whose origin remained uncertain after detailed clinical, radiologic, pathologic, and immunohistochemical evaluations. Six of these tumors (22\%) tested positive for albumin and were therefore reclassified as ICC. The remaining 21 cancers tested negative for albumin.

\section{DISCUSSION}

Intrahepatic cholangiocarcinoma presents a diagnostic dilemma for clinicians because no definitive immunohistochemical markers are available. However, patients cannot be treated without a definitive diagnosis. The liver is a preferred metastatic site for many different tumor types. If the primary site is not known, extensive immunohistochemical staining of the liver biopsy must be performed.

For ICC, no specific diagnostic marker exists. Therefore, patients undergo multiple invasive and noninvasive procedures to rule out a primary extra hepatic cancer. Often, patients undergo upper gastrointestinal endoscopies, colonoscopies, cervical pap smears, mammograms, positron emission tomography, computed tomography, and magnetic resonance imaging. These studies inflict a significant amount of stress on the patient, delay their care, and expose them to small but real risks of radiation and perforation. Thousands of dollars are spent on this extensive workup before the initiation of chemotherapy or surgical resection.

Patients and physicians urgently need a sensitive and specific biomarker for ICC. Albumin RNA ISH represents the solution to this clinical conundrum because it is a highly specific and sensitive marker for ICC (Fig. 4). Almost all ICCs (99\%) from two institutions tested positive for albumin, whereas adenocarcinomas that typically metastasize to the liver 
(gastrointestinal, pulmonary system, or breast adenocarcinomas) tested negative for albumin.

Albumin also represents a highly specific and sensitive marker for HCC. Whereas histologic evaluation typically cannot distinguish an ICC from metastatic adenocarcinoma, HCC generally can be distinguished from ICC with conventional histology and immunohistochemistry. In the current series, ICCs were distinguished from HCCs with certainty on an hemotoxylin and eosin (H\&E) stain. In cases with overlapping features, glypican 3, Hep Par 1, and arginase-1 were used. ${ }^{12,13}$

The presence of albumin also supports the hypothesis that ICC has greater similarity to HCC than to extrahepatic biliary carcinoma. Genetically engineered mouse models using an albumin-Cre system with liver-specific inactivation of $N f 2, M s t 1 / M s t 2$ develop HCC and ICC. ${ }^{12,14-16}$ A more recent model of ICC that closely resembles its human counterpart used an albumin-Cre-mediated somatic activation of $\mathrm{Kras}^{G 12 D}$ and a deletion of $p 53$ on histopathology. Although the majority of tumors were ICCs, HCCs also were identified. ${ }^{15}$ These data together with our data suggest that HCC and ICC arise from a common progenitor cell.

Unlike ICC and HCC, perihilar and distal bile duct carcinomas were uniformly negative for albumin. Although perihilar and distal bile duct carcinomas also are sometimes referred to as cholangiocarcinomas, recent data suggest that these tumors are genetically distinct from ICCs (e.g., mutations in Isocitrate dehydrogenase 1/2 (IDHI/2) are much more common in ICC). ${ }^{14-17}$

Our data also support the hypothesis that ICCs are biologically and genetically distinct from perihilar and extrahepatic bile duct carcinomas. Whereas ICCs are derived from a progenitor cell capable of hepatic and cholangiocytic differentiation, the progenitor cell of bile duct carcinoma is restricted to a cholangiocytic lineage.

Currently, the distinction between perihilar cholangio-carcinoma and ICC is based on the epicenter of the lesion, an often subjective exercise. We propose replacing this subjective paradigm with albumin RNA ISH because it is specific for ICC. Improved classification of these cancers based on molecular differences inherent to the cell of origin will improve our understanding of the cancer and also help in the designing of better cohorts for clinical trials.

Approximately 3-5\% of all cancers have no identifiable primary site. ${ }^{1,18,19}$ In this era of targeted therapy, making a definitive diagnosis has become even more important. Because albumin is sensitive and specific for ICC, it is a useful marker for evaluating carcinomas of unknown origin. In our series, $22 \%$ of the carcinomas of unknown origin tested positive for albumin.

In addition to $I D H I / 2$ mutations, genomic studies also have shown actionable recurrent translocation events involving the fibroblast growth factor receptor 2 (FGFR2) locus in ICCs. ${ }^{20}$ For patients with ICC rather than carcinoma of unknown origin, albumin RNA ISH would both limit the need for extensive radiologic and endoscopic evaluations and assist in 
the identification of tumors with actionable mutations that can be treated by targeted therapies. ${ }^{3,21}$

This study investigated whole tissue sections and tissue microarrays, confirming that RNA ISH can be performed on both surgically resected specimens and more limited tissue samples such as fine-needle aspirates and core biopsies. This novel technology cannot be replaced by an immunohistochemical assay for albumin due to the ubiquitous presence of albumin in both normal and neoplastic cells, making immunohistochemistry extremely difficult to interpret. Prior studies failed to detect albumin reliably in ICCs because they were unable to detect low abundance transcripts. 6,7

The branch-chain ISH platform amplifies the signal via a branched-chain technique. In addition to its high sensitivity, the platform also has a minimal nonspecific background signal compared with immunohistochemistry. Whereas RNA ISH has been used in the research laboratory for many decades, tissue-based RNA diagnostics have only recently been introduced. Our ability to detect albumin on an automated platform in virtually all ICCs with a minimal nonspecific signal is a significant advance compared with prior studies. 6,7

Gene expression profiling has been successfully used to locate a tumor's site of origin when histology and immunohistochemistry have been unable to make a diagnosis. ${ }^{22,23} \mathrm{~A}$ significant disadvantage of expression profiling is that the gene signature is an aggregate signal of tumor cells, stroma, and other mesenchymal elements that accompany the tumor, thus decreasing the sensitivity and specificity. ${ }^{23,24}$ The RNA ISH platform has a significant advantage because it preserves the morphologic detail of the tissue. Therefore, albumin staining can be localized to tumor or normal cells. Moreover, the branch-chain nucleic acid ISH assay is amenable to automation and hence could be adopted by most diagnostic clinical laboratories.

Albumin ISH provides a sensitive and specific marker for ICC. This test can be performed on a needle biopsy or a large specimen. Because the test is automated, it can be performed rapidly, allowing patients to forego numerous invasive and diagnostic tests. Albumin ISH should be used for all patients with a liver mass in which no primary malignancy was identified before they were subjected to extensive unnecessary testing.

\section{Supplementary Material}

Refer to Web version on PubMed Central for supplementary material.

\section{Acknowledgments}

We thank Dr. Gregory Lauwers for critically reviewing this manuscript and Dr. Elena Brachtel and Chin-Lee Wu for contributing tissue microarrays. This work was supported by the Howard Hughes Medical Institute (M.N.R.), the Burroughs Wellcome Trust (D.T.T., M.N.R.), K12CA087723-11A1 (D.T.T.), and Affymetrix, Inc. (D.T.T., M.N.R., V.D.). All the authors have read and approved this version of the manuscript. 


\section{References}

1. Everhart JE, Ruhl CE. Burden of Digestive Diseases in the United States Part III: liver, biliary tract, and pancreas. Gastroenterology. 2009; 136:1134-44. [PubMed: 19245868]

2. Blechacz B, Komuta M, Roskams T, Gores GJ. Clinical diagnosis and staging of cholangiocarcinoma. Nat Rev Gastroenterol Hepatol. 2011; 8:512-22. [PubMed: 21808282]

3. Khan SA, Davidson BR, Goldin RD, et al. Guidelines for the diagnosis and treatment of cholangiocarcinoma: an update. Gut. 2012; 61:1657-69. [PubMed: 22895392]

4. De Jong MC, Nathan H, Sotiropoulos GC, et al. Intrahepatic cholangiocarcinoma: an international multi-institutional analysis of prognostic factors and lymph node assessment. J Clin Oncol. 2011; 29:3140-5. [PubMed: 21730269]

5. Malaguarnera G, Paladina I, Giordano M, Malaguarnera M, Bertino G, Berretta M. Serum markers of intrahepatic cholangiocarcinoma. Dis Markers. 2013; 34:219-28. [PubMed: 23396291]

6. Tickoo SK, Zee SY, Obiekwe S, et al. Combined hepatocellular-cholangiocarcinoma: a histopathologic, immunohistochemical, and in situ hybridization study. Am J Surg Pathol. 2002; 26:989. [PubMed: 12170085]

7. Krishna M, Lloyd RV, Batts KP. Detection of albumin messenger RNA in hepatic and extrahepatic neoplasms: a marker of hepatocellular differentiation. Am J Surg Pathol. 1997; 21:147-52. [PubMed: 9042280]

8. Breborowicz J, Tamaoki T. Detection of messenger RNAs of alpha-fetoprotein and albumin in a human hepatoma cell line by in situ hybridization. Cancer Res. 1985; 45:1730-6. [PubMed: 2579734]

9. Yamaguchi K, Nalesnik MA, Carr BI. In situ hybridization of albumin mRNA in normal liver and liver tumors: identification of hepatocellular origin. Virchows Arch B Cell Pathol Incl Mol Pathol. 1993; 64:361-5. [PubMed: 8148956]

10. D'Errico A, Deleonardi G, Fiorentino M, Scoazec JY, Grigioni WF. Diagnostic implications of albumin messenger RNA detection and cytokeratin pattern in benign hepatic lesions and biliary cystadenocarcinoma. Diagn Mol Pathol. 1998; 7:289-94. [PubMed: 10207666]

11. Oliveira AMA, Erickson LAL, Burgart LJL, Lloyd RVR. Differentiation of primary and metastatic clear cell tumors in the liver by in situ hybridization for albumin messenger RNA. Am J Surg Pathol. 2000; 24:177-82. [PubMed: 10680884]

12. Zhou D, Conrad C, Xia F, et al. Mst1 and Mst2 maintain hepatocyte quiescence and suppress hepatocellular carcinoma development through inactivation of the Yap1 oncogene. Cancer Cell. 2009; 16:425-38. [PubMed: 19878874]

13. Yan BC, Gong C, Song J, et al. Arginase-1: a new immunohistochemical marker of hepatocytes and hepatocellular neoplasms. Am J Surg Pathol. 2010; 34:1147-54. [PubMed: 20661013]

14. Voss JS, Holtegaard LM, Kerr SE, et al. Molecular profiling of cholangiocarcinoma shows potential for targeted therapy treatment decisions. Hum Pathol. 2013; 44:1216-22. [PubMed: 23391413]

15. Wang P, Dong Q, Zhang C, et al. Mutations in isocitrate dehydrogenase 1 and 2 occur frequently in intrahepatic cholangiocarcinomas and share hypermethylation targets with glioblastomas. Oncogene. 2013; 32:3091-100. [PubMed: 22824796]

16. Kipp BR, Voss JS, Kerr SE, et al. Isocitrate dehydrogenase 1 and 2 mutations in cholangiocarcinoma. Hum Pathol. 2012; 43:1552-8. [PubMed: 22503487]

17. Borger DR, Tanabe KK, Fan KC, et al. Frequent mutation of isocitrate dehydrogenase (IDH)1 and IDH2 in cholangiocarcinoma identified through broad-based tumor genotyping. Oncologist. 2012; 17:72-9. [PubMed: 22180306]

18. Muir C. Cancer of unknown primary site. Cancer. 1995; 75(1 Suppl):353-6. [PubMed: 8001006]

19. Daud AI. Removing the unknown from the carcinoma of unknown primary. J Clin Oncol. 2013; 31:174-5. [PubMed: 23233703]

20. Borad MJ, Champion MD, Egan JB, et al. Integrated genomic characterization reveals novel, therapeutically relevant drug targets in FGFR and EGFR pathways in sporadic intrahepatic cholangiocarcinoma. PLoS Genet. 2014; 10:e1004135. [PubMed: 24550739] 
21. Xiao J-C, Ruck P, Adam A, Wang T-X, Kaiserling E. Small epithelial cells in human liver cirrhosis exhibit features of hepatic stem-like cells: immunohistochemical, electron microscopic and immunoelectron microscopic findings. Histopathology. 2003; 42:141-9. [PubMed: 12558746]

22. Hainsworth JD, Rubin MS, Spigel DR, et al. Molecular gene expression profiling to predict the tissue of origin and direct site-specific therapy in patients with carcinoma of unknown primary site: a prospective trial of the Sarah Cannon research institute. J Clin Oncol. 2013; 31:217-23. [PubMed: 23032625]

23. Monzon FA, Lyons-Weiler M, Buturovic LJ, et al. Multicenter validation of a 1,550-gene expression profile for identification of tumor tissue of origin. J Clin Oncol. 2009; 27:2503-8. [PubMed: 19332734]

24. Varadhachary G. New strategies for carcinoma of unknown primary: the role of tissue-of-origin molecular profiling. Clin Cancer Res. 2013; 19:4027-33. [PubMed: 23519898] 


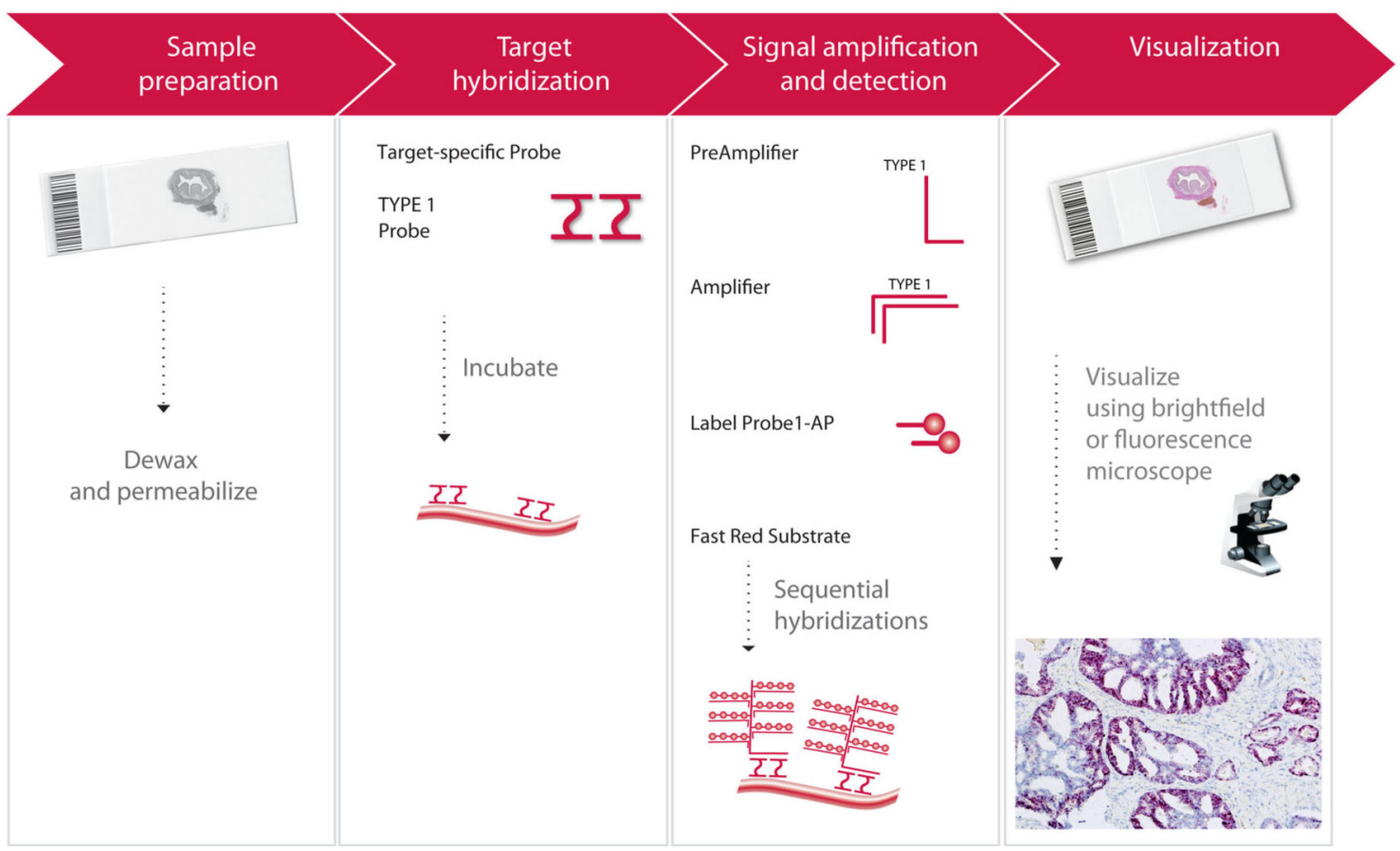

FIG. 1.

Cartoon schematic of RNA in situ hybridization (ISH) technology and methodology 


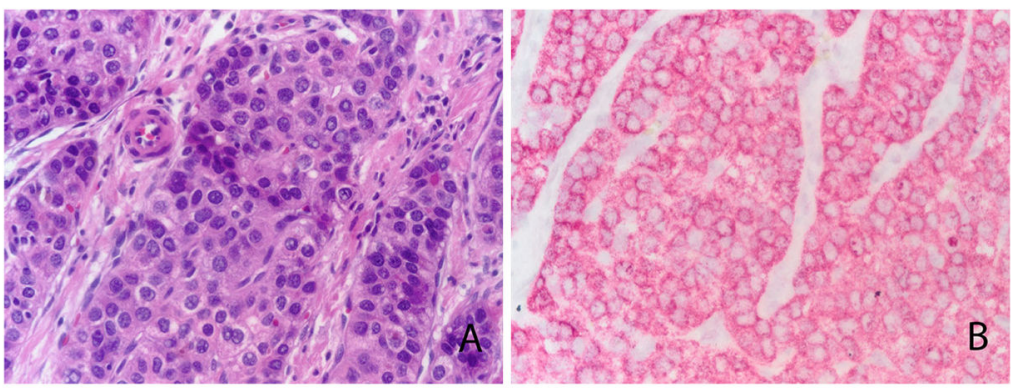

FIG. 2.

Hepatocellular carcinoma (HCC) diffuse and strongly positive for albumin. a Hematoxylin and eosin (H\&E) stain (left). b In situ hybridization (ISH) for albumin (right) 


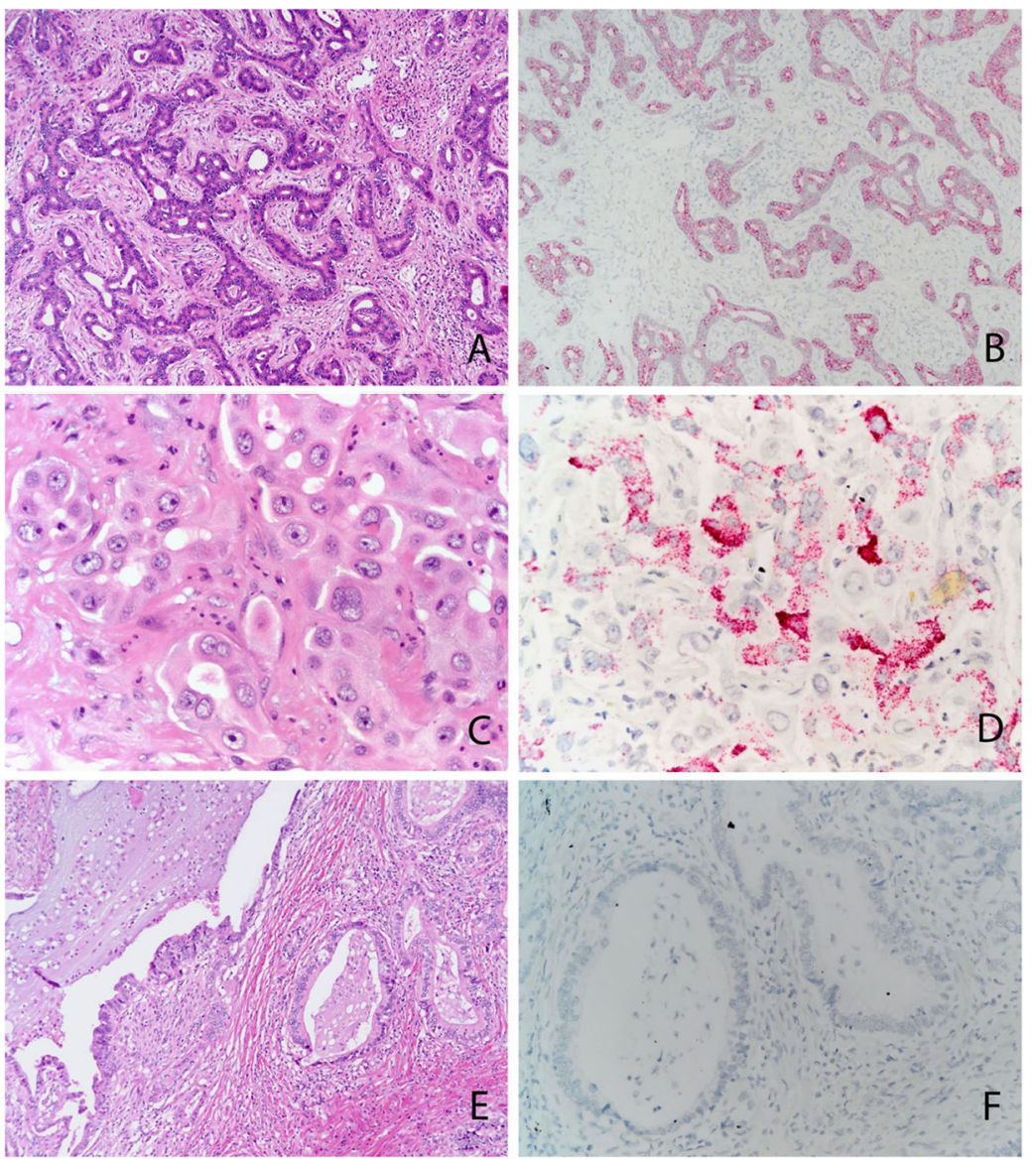

FIG. 3.

a, b Well-differentiated cholangiocarcinoma with diffuse reactivity for albumin. c, $\mathbf{d}$ Poorly differentiated cholangiocarcinoma with reactivity for albumin. e, f Perihilar bile duct carcinoma that tests negative for albumin. a, c, e Hematoxylin and eosin (H\&E) stain. b, d, f In situ hybridization (ISH) for albumin 


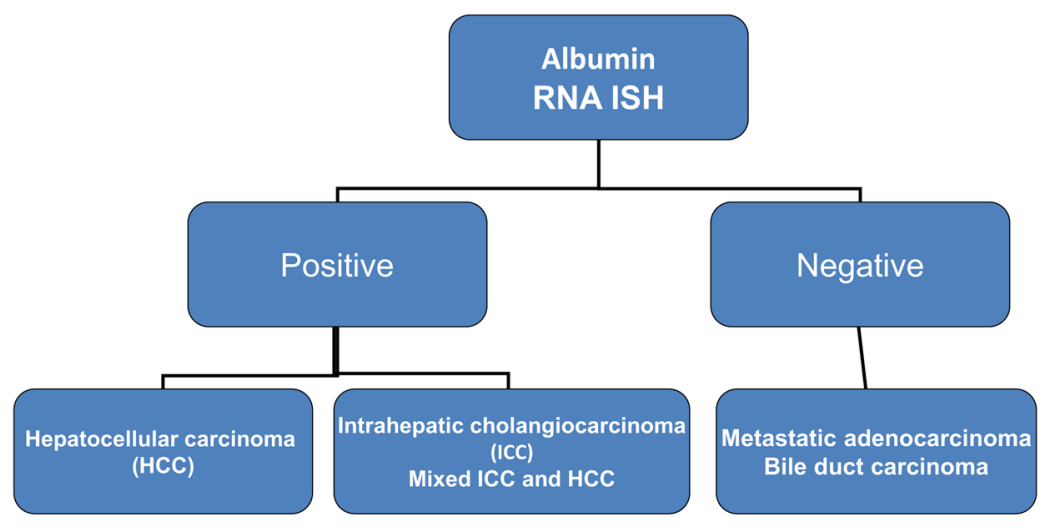

FIG. 4.

Proposed diagnostic algorithm for evaluation of hepatic neoplasms 